\title{
On the Security of Hash Functions Employing Blockcipher Postprocessing
}

\author{
Donghoon Chang $^{1}$, Mridul Nandi ${ }^{2}$, and Moti Yung ${ }^{3}$ \\ 1 National Institute of Standards and Technology, USA \\ 2 C.R. Rao AIMSCS, Hyderabad, India \\ 3 Google Inc. and Department of Computer Science, \\ Columbia University, New York, USA \\ \{pointchang, mridul.nandi\}@gmail.com, \\ my123@columbia.edu
}

\begin{abstract}
Analyzing desired generic properties of hash functions is an important current area in cryptography. For example, in Eurocrypt 2009, Dodis, Ristenpart and Shrimpton [8] introduced the elegant notion of "Preimage Awareness" (PrA) of a hash function $H^{P}$, and they showed that a PrA hash function followed by an output transformation modeled to be a FIL (fixed input length) random oracle is PRO (pseudorandom oracle) i.e. indifferentiable from a VIL (variable input length) random oracle. We observe that for recent practices in designing hash function (e.g. SHA-3 candidates) most output transformations are based on permutation(s) or blockcipher(s), which are not PRO. Thus, a natural question is how the notion of PrA can be employed directly with these types of more prevalent output transformations? We consider the Davies-Meyer's type output transformation $O T(x):=E(x) \oplus x$ where $E$ is an ideal permutation. We prove that $O T\left(H^{P}(\cdot)\right)$ is PRO if $H^{P}$ is $\operatorname{Pr} A$, preimage resistant and computable message aware (a related but not redundant notion, needed in the analysis that we introduce in the paper). The similar result is also obtained for $12 \mathrm{PGV}$ output transformations. We also observe that some popular double block length output transformations can not be employed as output transformation.
\end{abstract}

Keywords: PrA, PRO, PRP, Computable Message Awareness.

\section{Introduction}

Understanding what construction strategy has a chance to be a good hash function is extremely challenging. Further, nowadays it is becoming more important due to the current SHA3 competition which is intended to make a new standard for hash functions. In TCC'04, Maurer et al. [17] introduced the notion of indifferentiability as a generalization of the concept of the indistinguishability of two systems [16]. Indifferentiable from a VIL (variable input length) random oracle (also known as PRO or pseudorandom oracle) is the appropriate notion of random oracle for a hash-design. Recently, Dodis, Ristenpart and Shrimpton [8] introduced a generic method to show indifferentiable or PRO security proof 
of a hash function, whose final output function $\mathcal{R}$ is a FIL (fixed input length) random oracle. More precisely, they defined a new security notion of hash function, called preimage awareness (PrA), and showed that $F(M)=\mathcal{R}\left(H^{P}(M)\right)$ is PRO provided $H^{P}$ is preimage aware (supposed to be a weaker assumption). The result is applied to prove the indifferentiable security of the Skein hash algorithm [2], a second round SHA3 candidate. Informally, a hash function $H^{P}$ is called $\operatorname{PrA}$ if the following is true for any adversary $A$ having access to $P$ : For any $y$ committed by $A$, if a preimage of $y$ is not efficiently "computable" (by an algorithm called extractor) from the tuple of all query-responses of $P$ (called advise string) then $A$ should not be able to compute it even after making additional $P$ queries. This new notion seems to be quite powerful whenever we have composition of a VIL hash function and a FIL output transformation.

Our Result. We start with a preliminary discussion about the different notions and interrelationship among them. We note that there are hash functions whose final output transformation cannot be viewed as a random oracle e.g. some SHA3 second round candidates. So one needs to extended results beyond that of Dodis et al. to cover the cases of hash functions with various output transformations which are in use and this becomes our major objective, since it is important to assure good behavior of these hash functions as well. As a good example of a prevalent transform for construction of hash functions, we choose DaviesMeyer [21] $O T(x)=E(x) \oplus x$ where $E$ is a random permutation and study it in Section 3. We observe that the preimage awareness of $H^{P}$ is not sufficient for the PRO security of $F$. In addition to $\operatorname{PrA}$, if $H^{P}$ is also preimage resistant (PI) and computable message aware (as we define in Section [3.1), then $F^{P, E}$ is PRO (proved in Theorem 1). Informally speaking, a hash function $H^{P}$ is called computable message aware (or CMA) if there exists an efficient extractor (called computable message extractor) which can list the set of all computable messages whose $H^{P}$ outputs are implied with high probability given the advise string of $P$. The main difference with PrA is that here, no adversary is involved and the extractor does not get any specific target (see Definition 2). We show that both preimage resistant and CMA are not implied by PrA and hence these properties can not be ignored. Our result can then be employed to prove that a close variant of Grøstl is PRO (see Section 4) 1 . We continue our research in finding other good output transformations. We found 12 out of $20 \mathrm{PGVs}$ can be employed as output transformation $O T$ and we require similar properties of $H^{P}$, i.e. PrA, PI and CMA, to have PRO property of $O T\left(H^{P}\right)$ (see Section 5). However these three properties are not sufficient for some DBL post processors. In section 6] we show PRO attacks when some popular double block length post processors are employed. It would be an interesting future research work to characterize the properties of the inner hash function $H^{P}$ and the output transformation $O T$ such that $O T\left(H^{P}\right)$ become PRO. In the appendix we review the results of 89 .

$\overline{1}$ The indifferentiable security analysis of Grøstl has been studied in [1]. 


\section{Preliminaries}

A game is a tuple of probabilistic stateful oracles $G=\left(\mathcal{O}_{1}, \ldots, \mathcal{O}_{r}\right)$ where states can be shared by the oracles. The oracles can have access to primitives (e.g. random oracle) via black-box modes. It is reasonable to assume that all random sources of games come from the primitives. A probabilistic oracle algorithm $A$ (e.g. an adversary) executes with an input $x$, its oracle queries being answered by the corresponding oracles of $G$. Finally it returns $y:=A^{G}(x)$. An adversary $A$ may be limited by different resources such as its runtime, number of queries to different oracles, size of its inputs or outputs, etc. If $\theta$ is a tuple of parameters describing the available resources of $A$ then we say that $A$ is a $\theta$-adversary. In this paper $H^{P}$ is an $n$-bit hash function defined over a message space $\mathcal{M}$ based on a primitive $P$ which can be only accessed via a black-box.

Indifferentiability. The security notion of indifferentiability or PRO was introduced by Maurer et al. in TCC'04 [17. In Crypto'05, Coron et al. adopted it as a security notion for hash functions [6]. Let $F$ be a hash function based on ideal primitives $P=\left(P_{1}, \ldots, P_{j}\right)$ and $\mathcal{F}$ be a VIL random oracle, let $S^{\mathcal{F}}=$ $\left(S_{1}^{\mathcal{F}}, \ldots, S_{j}^{\mathcal{F}}\right)$ be a simulator (aimed to simulate $P=\left(P_{1}, \ldots, P_{j}\right)$ ) with access to $\mathcal{F}$, where $S_{i}$ 's can communicate to each other. Then, for any adversary $A$, the indifferentiability- or PRO-advantage of $A$ is defined by $\mathbf{A d}_{\mathbf{d v}_{F^{P}, S^{\mathcal{F}}}}^{\text {pro }}(A)=$ $\left|\operatorname{Pr}\left[A^{F, P}=1\right]-\operatorname{Pr}\left[A^{\mathcal{F}, S}=1\right]\right|$. When the value of the above advantage is negligible, we say that the hash function $F$ is indifferentiable or PRO. Maurer et al. [17] also proved that if $F$ is indifferentiable, then $\mathcal{F}$ (a VIL random oracle) used in any secure cryptosystem can be replaced by $F^{P_{1}, \ldots, P_{j}}$ with a negligible loss of security. In other words, $F$ can be used as a VIL PRO.

Preimage-Awareness or PrA. Dodis, Ristenpart and Shrimpton defined a new security notion called Preimage-Awareness (or PrA) for hash functions [89] which plays an important role in analyzing indifferentiability of a hash function 2. Given a game $G^{P}$ (can be $P$ itself), a tuple $\alpha=\left(\left(x_{1}, w_{1}\right), \ldots\right.$, $\left.\left(x_{s}, w_{s}\right)\right)$ is called an advise string at some point of time in the execution of $A^{G}$, if $w_{i}$ 's are responses of all $P$-queries $x_{i}$ 's until that point of time. A PrA $(q, e, t)$ adversary $A$ (making $q$ queries and running in time $t$ ) commits $y_{1}, \ldots, y_{e}$ during the execution of $A^{P}$ and finally returns $M$. We write $\left(y_{1}, \ldots, y_{e}\right) \leftarrow A_{\text {guess }}^{P}$, $M \leftarrow A^{P}$ and denote the advise string at the time $A_{\text {guess }}^{P}$ commits $y_{i}$ by $\alpha_{i}$. The guesses and $P$-queries can be made in any order.

Definition 1. The PrA-advantage of $H^{P}$ with an extractor $\mathcal{E}$ is defined as $\mathbf{A} \mathbf{d} \mathbf{v}_{H, P, \mathcal{E}}^{\mathrm{pra}}(q, e, t)=\max _{A} \mathbf{A d}_{H, P, \mathcal{E}}^{\mathrm{pra}}(A)$ where maximum is taken over all $(q, e, t)$ adversaries $A$ and the $\operatorname{Pr} A$ advantage of $A$, denoted $\mathbf{A d v}_{H, P, \mathcal{E}}^{\mathrm{pra}}(A)$, is defined as

$$
\operatorname{Pr}\left[\exists i, H^{P}(M)=y_{i}, M \neq \mathcal{E}\left(y_{i}, \alpha_{i}\right): M \leftarrow A^{P} ;\left(y_{1}, \ldots, y_{e}\right) \leftarrow A_{\text {guess }}^{P}\right] .
$$

$H^{P}$ is called $\left(q, e, t, t_{e}, \epsilon\right)-\operatorname{PrA}$ if $\mathbf{A} \mathbf{d v}_{H, P, \mathcal{E}}^{\text {pra }}(q, e, t) \leq \epsilon$ for some extractor $\mathcal{E}$ with runtime $t_{e}$. In short, we say that a hash function is $\operatorname{PrA}$ or preimage-aware if there exists an "efficient" extractor such that for all "reasonable" adversaries $A$ the PrA advantage is "small". 
Implication among Collision Resistant, Preimage resistant (PI), PrA. If a collision attacker $B^{P}$ returns a collision pair $\left(M, M^{\prime}\right)$, then a PrA attacker makes all necessary $P$ queries to compute $H^{P}(M)=H^{P}\left(M^{\prime}\right)=y$ and finally returns $M$ if $\mathcal{E}(y, \alpha)=M^{\prime}$, o.w. returns $M^{\prime}$. So a PrA hash function must be collision resistant. In [8] the authors consider a weaker version of $\operatorname{PrA}$ (called weak-PrA) where an extractor can return a set of messages (possibly empty) whose output is $y$. A PrA adversary wins this new weak game if it can find a preimage of $y$ different from those given by the extractor. They also have shown that $\operatorname{PrA}$ is equivalent to collision resistant and weak-PrA. One can modify a definition of a preimage-resistant hash function by introducing only one collision pair. It still remains preimage resistant as the randomly chosen target in the particular collision value has negligible probability. However, it is not preimageaware since a collision is known. On the other hand $H^{P}(x)=P^{-1}(x)$ or $H^{P}(x)=$ $x$ are not preimage resistant but PrA.

\section{Hash Function with Output Transformation $E(x) \oplus x$}

In [8, hash functions have been analyzed for which the output transformation can be modeled as a FIL random oracle. Generally, we can consider various kinds of output transformations such as Davis-Meyer, PGV compression functions [20] or some DBL (Double Block Length) compression functions 1912]1315 in the ideal cipher model. Traditionally, the most popular known design of hash function uses one of the above post-processors. It is well-known that all such compression functions are not indifferentiably secure [6]. So, we need a separate analysis from [8. In this section, we consider Davis-Meyer transformation $O T(x)=E(x) \oplus x$, where $E$ is a permutation modeled as a "random permutation." A simple example of $H^{P}$ (e.g. the identity function) tells us that the preimage awareness is not a sufficient condition to have PRO after employing Davis-Meyer post-processor. This suggests that we need something stronger than PrA. We first observe that the preimage attack on identity function can be exploited to have the PRO attack. So preimage resistant can be a necessary condition. We define a variant of preimage resistant, called multipoint-preimage (or $\mathrm{mPI}$ ), which is actually equivalent to PI. The multipoint-preimage (or $\mathrm{mPI}$ ) advantage of a $(q, t, s)$-adversary $A$ (i.e., adversary which makes $q$ queries, runs in time $t$ and has $s$ targets) for $H^{P}$ is defined as

$$
\mathbf{A d v}_{H^{P}}^{\mathrm{mPI}}(A)=\operatorname{Pr}_{h_{1}, \ldots, h_{s} \stackrel{\leftrightarrow}{\leftarrow}\{0,1\}^{n}}\left[\exists i, H^{P}(M)=h_{i}: M \leftarrow A^{P}\left(h_{1}, \ldots, h_{s}\right)\right]
$$

When $s=1$, it corresponds to the classical preimage advantage $\operatorname{Adv}_{H^{P}}^{\mathrm{PI}}(A)$. Conversely, mPI advantage can be bounded above by preimage advantage as described in the following. For any $(q, t, s)$-adversary $A$ with multipoint-preimage advantage $\epsilon$ against $H^{P}$, there is a $(q, t+O(s))$-adversary $A^{\prime}$ with preimageadvantage $\epsilon / s$. The adversary $A^{\prime}$ generates $(s-1)$ targets randomly and embeds his target among these in a random position. So whenever an mPI adversary $A$ finds a multipoint preimage of these $s$ targets, it is the preimage of $A$ 's target 
with probability $1 / s$ (since there is no way for $A$ to know the position of the target for $A^{\prime}$ ). W.l.o.g. one can assume that the targets are distinct and chosen at random. Otherwise we remove all repeated $h_{i}$ 's and replace them by some other random distinct targets. So we have the following result.

Lemma 1. Let $h_{1}, \ldots h_{s}$ be distinct elements chosen at random (i.e. outputs of a random permutation for distinct inputs). Then, any $(q, t)$-adversary $A^{P}$ can find one of the preimages of $h_{i}$ 's with probability at most $s \times \mathbf{A d v}_{H^{P}}^{\mathrm{PI}}(q, t)$.

\subsection{Computability}

Next we show that preimage resistant and $\operatorname{PrA}$ are not sufficient to prove the PRO property. Consider the following example based on an $n$-bit one-way permutation $f$ and random oracle $P$.

Example 1. $H^{P}(m)=P(f(m)) \oplus m$. Given $\alpha=(f(m), w)$ it is hard to find $m$ and hence there is no efficient extractor to find the message $m$ even though an adversary $A$ knows $m$ and its $H^{P}$-output. An adversary can compute $z=\mathcal{F}(m)$ and makes $E^{-1}(z \oplus w \oplus m)$ query. No feasible simulator can find message $m$ from it with non-negligible probability and hence cannot return $w \oplus m$. However $w \oplus m$ is the response when $A$ interacts with the real situation $\left(F^{P, E}, P, E, E^{-1}\right)$. So $A$ can have a PRO attack to $F$. It is easy to see that $H^{P}$ is preimage resistant and $\operatorname{PrA}$ (given the advise string $\alpha=\left(\left(x_{1}, w_{1}\right), \ldots,\left(x_{q}, w_{q}\right)\right)$ and the target $x$ (that helps to find $m$ back) the extractor finds $i$ for which $f\left(w_{i} \oplus x\right)=x_{i}$ and then returns $\left.w_{i} \oplus x\right)$.

The above example motivates us to define a computable message given an advise string. A message $M$ is called computable from $\alpha$ if there exists $y$ such that $\operatorname{Pr}\left[H^{P}(M)=y \mid \alpha\right]=1$. In other words, the computation of $H^{P}(M)=y$ can be made without making any further $P$-queries. We require the existence of an efficient extractor $\mathcal{E}_{\text {comp }}$, called computable message extractor, which can list all computable messages given the advise string. We note that this is not same as weak-PrA as the extractor has to find all messages whose outputs can be computed to a value (unlike $\operatorname{PrA}$, no such fixed target is given here). This notion does not involve any adversary.

Definition 2. A pair $\left(H^{P}, \mathcal{E}_{\text {comp }}\right)$ is called $\left(q, q_{H}, \epsilon\right)$-computable message aware or CMA if for any advise string $\alpha$ with $q$ pairs, the number of computable messages is at most $q_{H}$ and $\mathcal{E}_{\mathrm{comp}}(\alpha)$ outputs all these. Moreover, for any noncomputable messages $M, \operatorname{Pr}\left[H^{P}(M)=y \mid \alpha\right] \leq \epsilon, \forall y$.

A hash function $H^{P}$ is called $\left(q, q_{H}, \epsilon, t_{c}\right)$-computable message aware or CMA if there is $\mathcal{E}_{\text {comp }}$ with run time $t_{c}$ such that $\left(H^{P}, \mathcal{E}_{\text {comp }}\right)$ is $\left(q, q_{H}, \epsilon\right)$-CMA. In short we say that $H^{P}$ is $\mathrm{CMA}$ if it is $\left(q, q_{H}, \epsilon, t_{c}\right)$-computable message aware where for any feasible $q, q_{H}$ and $t_{c}$ are feasible and $\epsilon$ is negligible. We reconsider the above example $H^{P}(m)=P(f(m)) \oplus m$ for an one-way permutation $f$. We have seen that it is both PI and PrA. However, there is no efficient extractor that can not find all computable messages given the advise string say $(f(m), w)$. In fact, 
$m$ is computable but there is no way to know it by the extractor only from the advise string (extractor can know if the target $f(m) \oplus w=H^{P}(m)$ is given).

To be a computable message aware, the list of computable message has to be small or feasible so that an efficient computable message extractor can exist. For example, the identity function has a huge set of computable messages given any advise string which cannot be listed by any efficient algorithm even though we theoretically know all these messages.

\subsection{PRO Analysis of a Hash Function with OT $E(x) \oplus x$}

In this section we prove that $O T\left(H^{P}()\right)$ is PRO whenever $H^{P}$ is PrA, PI and CMA. We give an informal idea how the proof works. Note that for $E$-query, $E(x) \oplus x$ almost behaves like a random oracle and hence PrA property of $H^{P}$ takes care the simulation. This would be similar to the random oracle case except that we have to deal with the fact there is no collision on $E$. The simulation of responses of $P$-queries will be same as $P$. The non-trivial part is to response $E^{-1}$ query. If the $E^{-1}$-query $y$ is actually obtained by $y=\mathcal{F}(M) \oplus H^{P}(M)$ then simulator has to find the $M$ to give a correct response. Since simulator has no idea about the $\mathcal{F}(M)$ as he can not see $\mathcal{F}$-queries, the query $y$ is completely random to him. However, he can list all computable messages and try to compute $H^{P}(M)$ and $\mathcal{F}(M)$. This is why we need CMA property. The simulator should be able to list all computable messages only from the $P$ query-responses. If he finds no such messages then he can response randomly. The simulator would be safe as long as there is no preimage attack to the random output. Now we provide a more formal proof.

Let $F^{P, E}(M)=E\left(H^{P}(M)\right) \oplus H^{P}(M)$ and $A$ be a PRO adversary making at $\operatorname{most}\left(q_{0}, q_{1}, q_{2}, q_{3}\right)$ queries to its four oracles with bit-size $l_{\max }$ for the longest $\mathcal{O}_{0^{-}}$ query. We assume that $H^{P}(\cdot)$ is preimage resistant and $\left(q^{*}, q_{H}, \epsilon\right)$-computable message aware for an efficient computable message extractor $\mathcal{E}_{\text {comp }}$ where $q^{*}=$ $q_{1}+q_{2} \mathrm{NQ}\left[l_{\text {max }}\right]$. Let $q=q_{H}+q^{\prime}$ and $q^{\prime}=q_{0}+q_{1}+q_{2}+q_{3}$. For any given PrA-extractor $\mathcal{E}$, we can construct a simulator $S^{\mathcal{F}}=\left(S_{1}, S_{2}, S_{3}\right)$ (defined in the oracles of $C_{A}$ in Fig. 3) that runs in time $t^{*}=O\left(q^{2}+q_{3} \operatorname{Time}\left(\mathcal{E}_{\text {comp }}\right)\right)$. Given any indifferentiability adversary $A$ making at most $\left(q_{0}, q_{1}, q_{2}, q_{3}\right)$ queries to its four oracles with bit-size $l_{\max }$ for the longest $\mathcal{O}_{0}$-query, there exists a $\operatorname{PrA}\left(q, q_{2}+1, t\right)$-adversary $C_{A}$ with runtime $t=\operatorname{Time}(A)+O\left(q_{2} \cdot \operatorname{Time}(\mathcal{E})+\right.$ $\left.q_{0}+q_{1}+\left(q_{2}+q_{0}\right) \mathrm{NQ}\left[l_{\max }\right]\right)$. Now we state two lemmas which are useful to prove the main theorem of the section. Proof ideas of these lemmas are very similar to that of Lemma 8 and Lemma 10. The games $G 4$ and $G 5$ are defined in the Fig. 2. We use a simulation oracle simE which works given a runtime database E. A random element from the set $\{0,1\}^{n} \backslash \operatorname{Range}(\mathrm{E})$ is returned for $\operatorname{simE}[1, y]$ whenever $y$ is not in the domain of E. Similarly a random element from the set $\{0,1\}^{n} \backslash \operatorname{Domain}(\mathrm{E})$ is returned in $\operatorname{sim} E[-1, c]$ whenever $c$ is not in the range of $\mathrm{E}$. Whenever $y$ or $c$ are defined before the simulator oracle just returns the previous returned value. We use three such simulation oracles for $\mathrm{E}_{0}$ (which keeps the input output behavior of $\mathrm{E}$ due to $\mathcal{O}_{0}$ queries only), $\mathrm{E}_{1}$ 


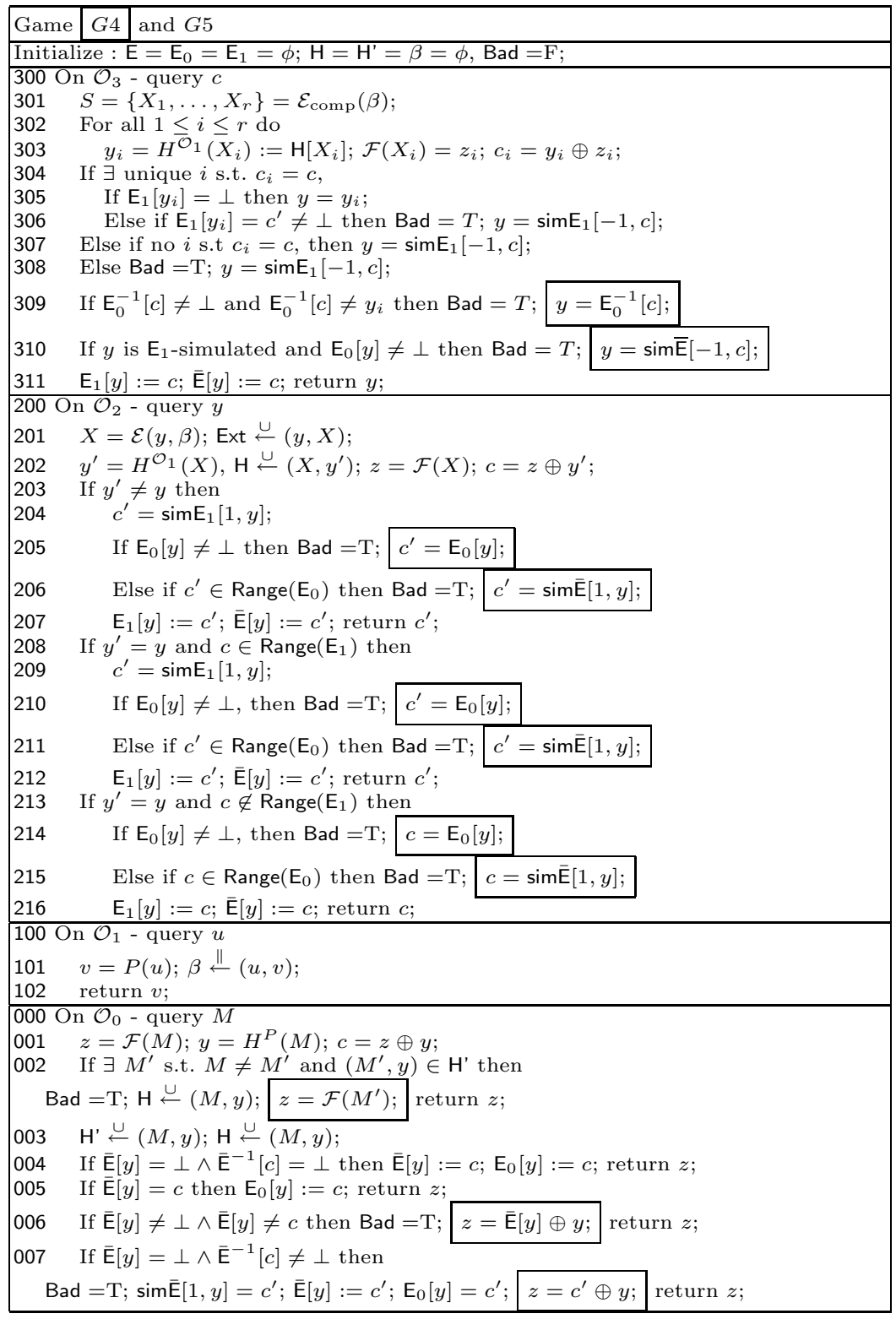

Fig. 1. $G 4$ executes with boxed statements whereas $G 5$ executes without these. $G_{4}$ and $G_{5}$ perfectly simulate $\left(F^{P, E}, P, E, E^{-1}\right)$ and $\left(\mathcal{F}, S_{1}, S_{2}, S_{3}\right)$, respectively. Clearly $G 4$ and $G 5$ are identical-until-Bad. 
(which keeps the input output behavior of $\mathrm{E}$ due to $\mathcal{O}_{2}$ and $\mathcal{O}_{3}$ queries) and $\overline{\mathrm{E}}$ (which keeps the input output behavior of $E$ for all queries, i.e. it is the union of the previous two unions).

\section{Lemma 2.}

$G 4 \equiv\left(F^{P, E}, P, E, E^{-1}\right), G 5 \equiv\left(\mathcal{F}, S_{1}, S_{2}, S_{3}\right)$ and $G 4, G 5$ are identical-untilBad.

Proof. It is easy to see from the pseudocode that $G 4, G 5$ are identical-untilBad. The games $G 5$ and the oracles simulated by $C_{A}$ (same as $\left(\mathcal{F}, S_{1}, S_{2}, S_{3}\right)$ ) are actually identical. They have common random sources which are namely $\mathcal{F}$, $P$ and the simulated oracle $\operatorname{sim}_{0}$ (we can ignore the dead conditional statements which have boxed statements which are not actually executed in game $G 5$ ). Now it remains to show that $G 5$ is equivalent to a real game for a PRO attacker. Note that oracles $\mathcal{O}_{2}$ and $\mathcal{O}_{3}$ are statistically equivalent to a random permutation $\overline{\mathrm{E}}$ and its inverse which are simulated runtime. Moreover $\mathcal{O}_{0}$ returns $\mathcal{F}(M)$ if $\overline{\mathrm{E}}[y], \overline{\mathrm{E}}^{-1}[c]$ are undefined or $\overline{\mathrm{E}}[y]=c$ where $y=H^{P}(M)$ and $c=\mathcal{F}(M) \oplus y$. In all other cases $\mathcal{O}_{0}(M)$ either computes or simulates $c^{\prime}=\overline{\mathrm{E}}[y]$ and returns $c^{\prime} \oplus y$. So $\mathcal{O}_{0}(M)$ is statistically equivalent to the oracle $\overline{\mathrm{E}}\left(H^{P}()\right) \oplus H^{P}()$. Hence $G 4$ is statistically equivalent to $\left(F^{P, E}, P, E, E^{-1}\right)$.

The following result follows immediately from the fact that $\mathcal{F}$ and $H^{P}$ are statistically independent and $\mathcal{F}$ is a random oracle.

Lemma 3. For any adversary $C^{P, \mathcal{F}}$ making q queries to the $n$-bit random oracle $\mathcal{F}$ we have $\operatorname{Pr}\left[\mathcal{F}(M) \oplus H^{P}(M)=\mathcal{F}\left(M^{\prime}\right) \oplus H^{P}\left(M^{\prime}\right), M \neq M^{\prime}:\left(M, M^{\prime}\right) \leftarrow C\right] \leq$ $q(q-1) / 2^{n+1}$.

Lemma 4. Whenever $A^{G 5}$ sets Bad true, $C_{A}$ also sets one of the Bad events true. Moreover,

$$
\begin{gathered}
\operatorname{Pr}\left[C_{A} \text { sets Bad true }\right] \leq \mathbf{A d v}_{H^{P}, P, \mathcal{E}}^{\mathrm{pra}}\left(C_{A}\right)+q_{3} \times \mathbf{A d v}_{H^{P}}^{\mathrm{PI}}(q, t)+ \\
q_{0} q_{3} \epsilon+\frac{2 q_{0} q_{3}+q_{2} q_{0}}{2^{n}-q_{0}-q_{2}-q_{3}}+\frac{\left(q_{H}+q_{2}+q_{0}\right)^{2}}{2^{n+1}} .
\end{gathered}
$$

Proof. The first part of the lemma is straightforward and needs to be verified case by case. We leave the details for readers to verify. It is easy to see that whenever Bad $_{\text {pra }}$ sets true $C$ is successful in a PrA attack. Now we estimate the probability of the other bad events, from which the theorem follows.

1. $\operatorname{Pr}\left[\operatorname{Bad}_{m P I}=T\right] \leq q_{3} \times \operatorname{Addv}_{H^{P}}^{P I}\left(q^{*}, t^{*}\right)$. It is easy to see that whenever $\operatorname{Bad}_{m P I}$ sets true we have a preimage of some $y_{i}$ which is generated from $\operatorname{sim} E_{1}$. Note that $\operatorname{sim} E_{1}$ responds exactly like a random permutation. So by lemma 1 we have the bound.

2. $\operatorname{Pr}\left[\operatorname{Bad}_{c o m p}=T\right] \leq q_{0} q_{3} \epsilon$. Whenever $\operatorname{Bad}_{\text {comp }}$ sets true we should have $H^{P}\left(M_{i}\right)=y_{i}$ where $M_{i}$ is not computable (since it is not in the list given by $\left.\mathcal{E}_{\text {comp }}\right)$. So from computable message awareness definition we know that $\operatorname{Pr}\left[H^{P}\left(M_{i}\right)=y_{i}\right] \leq \epsilon$. The number of such $M_{i}$ 's and $y_{i}$ 's are at most $q_{0} q_{3}$. 


\begin{tabular}{|c|c|}
\hline The Oracles $\mathcal{O}_{2}\left(\right.$ or $\left.S_{2}\right)$ and $\mathcal{O}_{3}\left(\right.$ or $\left.S_{3}\right)$ & $\begin{array}{l}\text { The oracles } \mathcal{O}_{0}(\text { or } \mathcal{F}), \mathcal{O}_{1}(\text { or } P) \text { and } \\
\text { Finalization }\end{array}$ \\
\hline $\begin{array}{l}\text { /The VIL random oracle } \mathcal{F} \text { is simulated by } \\
C_{A} / \\
\text { Initialize }: \mathrm{E}_{1}=\mathcal{L}=\mathcal{L}_{1}=\mathrm{F}^{\prime}=\mathrm{H}=\beta= \\
\phi ;\end{array}$ & \begin{tabular}{|l}
$\mid$ The VIL random oracle $\mathcal{F}$ is simulated \\
by $C_{A} /$ \\
100 On $\mathcal{O}_{1}-$ query $u$
\end{tabular} \\
\hline $\begin{array}{l}\text { Run } A \text { and response its oracles } \\
300 \mathrm{On} \mathcal{O}_{3}-\text { query } c\end{array}$ & $101 \quad v=P(u) ; \beta \stackrel{\|}{\leftarrow}(u, v) ;$ return $v$; \\
\hline $301 \quad S=\left\{X_{1}, \ldots, X_{r}\right\}=\mathcal{E}_{\mathrm{comp}}(\beta)$ & 000 On $\mathcal{O}_{0}($ or $\mathcal{F})$ - query $M$ \\
\hline $\begin{array}{l}302 \quad \text { For all } 1 \leq i \leq r \text { do } \\
303 \quad y_{i}=H^{\mathcal{O}_{1}}\left(X_{i}\right):=\mathrm{H}\left[X_{i}\right] ; \mathcal{F}\left(X_{i}\right)= \\
z_{i} ;\end{array}$ & $z=\mathcal{F}(M) ; \mathcal{L} \longleftarrow M$ \\
\hline $\begin{array}{l}304 \quad c_{i}=y_{i} \oplus z_{i} ; \mathrm{F}^{\prime}\left[X_{i}\right]=c_{i} ; \mathcal{L}_{1} \stackrel{\cup}{\longleftarrow} \text {; } \\
305 \quad \text { If } \exists \text { unique } i, c_{i}=c, \\
306 \quad \text { If } \mathrm{E}_{1}\left[y_{i}\right]=\perp \text { then } y=y_{i}\end{array}$ & \begin{tabular}{|l} 
Finalization () \\
501 If collision in $\mathrm{F}^{\prime}$ then $\operatorname{Bad}_{F 1}=\mathrm{T} ;$ \\
502 If collision in $\mathrm{H}$ then $\operatorname{Bad}_{\operatorname{PrA}}=\mathrm{T}$ \\
Finish ()$;$
\end{tabular} \\
\hline $\begin{array}{l}\text { Else if } \mathcal{O}_{3}\left(c^{\prime}\right)=y_{i} \text { was queried and } \\
\text { no } i \text { on that query then } \\
\quad \operatorname{Bad}_{P I}=T ; y=\operatorname{simE}_{1}[-1, c]\end{array}$ & $\begin{array}{l}503 \text { For all } M \in \mathcal{L} \text { do 504-518 } \\
504 z=\mathcal{F}(M), \mathrm{H}[M]=H^{P}(M)=y \\
505 \mathrm{~F}^{\prime}[M]=c=y \oplus z ;\end{array}$ \\
\hline Else if no $i$ then $y=\operatorname{sim}_{1}[-1, c]$ & $\begin{array}{l}506 \text { If } \mathrm{F}^{\prime}[X]=c, X \neq M \text { then } \\
\operatorname{Bad}_{F 1}=\mathrm{T} ;\end{array}$ \\
\hline Else $\operatorname{Bad}_{F 1}=\mathrm{T} ; y=\operatorname{simE}_{1}[-1, c]$ & $\mid \begin{array}{l}507 \text { If } \mathrm{H}[X]=y, X \neq M \text { then } \\
\operatorname{Bad}_{\operatorname{Pr} A}=\mathrm{T} ; \operatorname{Finish}() ;\end{array}$ \\
\hline $\mathrm{E}_{1}[y]=c ;$ return $c$ & $\begin{array}{l}508 \text { If } \operatorname{Ext}[y] \neq \perp, M \text { then } \operatorname{Bad}_{\operatorname{Pr} A}=\mathrm{T} \\
\text { Finish(); } \\
509 \text { If } \mathcal{O}_{2}(y)=c_{i}, y \neq y_{i}\end{array}$ \\
\hline 00 On $\mathcal{O}_{2}$ - query $y:=y_{i}, i=$ & $\mid 510 \quad$ then $\operatorname{Bad}_{E 1}=\mathrm{T}$ \\
\hline$X=\mathcal{E}\left(y_{i}, \beta\right) ; \operatorname{Ext} \stackrel{\cup}{\longleftarrow}(y, X) ;$ & $\mid \begin{array}{l}511 \text { Else if } \mathcal{O}_{3}\left(c_{i}\right)=y \neq y_{i} \text { after } M_{i-} \\
\text { query }\end{array}$ \\
\hline $\begin{array}{l}202 y^{\prime}=H^{\mathcal{O}_{1}}(X), \mathrm{H} \stackrel{\cup}{\longleftarrow}\left(X, y^{\prime}\right) ; z= \\
\mathcal{F}(X) ;\end{array}$ & then $\operatorname{Bad}_{c o m p}=\mathrm{T}$; \\
\hline $203 \quad \mathcal{L}_{1} \stackrel{\cup}{\longleftarrow} X ; c=z \oplus y ; \mathrm{F}^{\prime}[X]=c ;$ & $\mid \begin{array}{l}513 \text { Else if } \mathcal{O}_{3}\left(c_{i}\right)=y \neq y_{i} \text { before } M_{i^{-}} \\
\text {query }\end{array}$ \\
\hline If $y^{\prime}$ & then $\operatorname{Bad}_{F 2}=\mathrm{T}$; \\
\hline then $c=\operatorname{simE}_{1}[1, y]$ & $\mid \begin{array}{l}515 \text { Else if } \mathcal{O}_{3}(c)=y_{i} \text { after } M_{i} \text {-query, } \\
c \neq c_{i}\end{array}$ \\
\hline If $y^{\prime}=y, \mathcal{O}_{3}(c)$ was queried & 516 then $\operatorname{Bad}_{E 2}=\mathrm{T}$ \\
\hline then $\operatorname{Bad}_{F 1}=\mathrm{T} ; c=\operatorname{sim}_{1}[1$ & $\| \begin{array}{l}517 \text { Else if } \mathcal{O}_{3}(c)=y_{i} \text { before } M_{i} \text {-query, } \\
c \neq c_{i}\end{array}$ \\
\hline $2(y)=c \mathrm{w}$ & then $\operatorname{Bad}_{P I}=\mathrm{T}$; \\
\hline 10 & 519 \\
\hline 10 & \\
\hline
\end{tabular}

Fig. 2. The oracles simulated by PrA adversary $C_{A}$ to response an PRO adversary $A$. It has a finalization procedure which also sets some bad event true. Finish() which is defined similarly as in Fig. 4 It mainly completes the PrA attack. It is easy to see that whenever Finish() is being executed either we have a collision in $H^{P}$ or there is some message $M$ such that $H^{P}(M)=y,(y, M) \notin$ Ext. 
3. All other bad events hold due to either the special outputs of $\mathcal{F}(M)$ (when $\operatorname{Bad}_{F 1}=T$ or $\operatorname{Bad}_{F 2}=T$, we apply the lemma 3) or the special outputs of $\operatorname{simE}(c)$ (when $\operatorname{Bad}_{E 1}=T$ and $\operatorname{Bad}_{E 2}=T$ ). One can show the following:

$$
\operatorname{Pr}\left[\operatorname{Bad}_{E 1 \vee E 2 \vee F 1 \vee F 2}=T\right] \leq \frac{2 q_{0} q_{3}+q_{2} q_{0}}{2^{n}-q_{0}-q_{2}-q_{3}}+\frac{\left(q_{H}+q_{2}+q_{0}\right)^{2}}{2^{n+1}} .
$$

We have used Lemma 3 to bound the bad event $\operatorname{Bad}_{F 1}$. The other bad event probability calculations are straightforward. We leave details to readers.

The main theorem of the section follows from the above lemmas.

Theorem 1. For any indifferentiability adversary $A$ making at most $\left(q_{0}, q_{1}, q_{2}\right.$, $\left.q_{3}\right)$ queries to its four oracles with bit-size $l_{\max }$ for the longest $\mathcal{O}_{0}$-query, there exists a $\operatorname{Pr} A\left(q, q_{2}+1, t\right)$-adversary $C_{A}$ with runtime $t=\operatorname{Time}(A)+O\left(q_{2}\right.$. $\left.\operatorname{Time}(\mathcal{E})+q_{0}+q_{1}+\left(q_{2}+q_{0}\right) N Q\left[l_{\text {max }}\right]\right)$ and

$$
\mathbf{A d v}_{F, S}^{\mathrm{pro}}(A) \leq \mathbf{A} \mathbf{d} \mathbf{v}_{H^{P}, P, \mathcal{E}}^{\mathrm{pra}}\left(C_{A}\right)+q_{3} \times \mathbf{A} \mathbf{d} \mathbf{v}_{H^{P}}^{\mathrm{PI}}(q, t)+\delta,
$$

where $\delta=q_{0} q_{3} \epsilon+\frac{2 q_{0} q_{3}+q_{2} q_{0}}{2^{n}-q_{0}-q_{2}-q_{3}}+\frac{\left(q_{H}+q_{2}+q_{0}\right)^{2}}{2^{n+1}}, H^{P}(\cdot)$ is preimage resistant and $\left(q^{*}, q_{H}, \epsilon\right)$-computable message aware for an efficient computable message extractor $\mathcal{E}_{\text {comp }}$ where $q^{*}=q_{1}+q_{2} N Q\left[l_{\max }\right]$.

\section{Application of Theorem 1: PRO Analysis of a Variant of Grøstl}

As an application of Theorem 1 we prove the PRO analysis of a variant of Grøstl hash function in which the output transformation is based on a permutation independent of the permutations used in iteration. The compression function $f^{P, Q}(z, m)=P(z \oplus m) \oplus Q(m) \oplus z$, where $P$ and $Q$ are invertible permutations on $n$-bit modeled to be independent random permutations (adversary can also have access to inverses). The hash function $H^{P, Q}$ of Grøstl without output transformation is Merkle-Damgård with strengthening (SMD) and the output transformation is $\operatorname{trunc}_{s}(P(x) \oplus x)$. In case of the variant of the hash function, the output transformation is same as the previous section, i.e. $O T(x)=E(x) \oplus x$ where $E$ is a random permutation independent with $P$ and $Q$. Since SMD preserves preimage awareness and preimage resistance of the underlying compression function, we focus on the proof of the compression function $f^{P, Q}$ to prove $\operatorname{PrA}$ and preimage resistance. The proof of the following lemmas are straightforward are given in the full version of the paper [5].

Lemma 5. For any advise string $\alpha_{P}$ and $\alpha_{Q}$ of sizes $\left(q_{1}+q_{2}\right)$ and $\left(q_{3}+q_{4}\right)$ (for $\left(P, P^{-1}\right)$ and $\left(Q, Q^{-1}\right)$ respectively) the number of computable messages is at most $q_{f} \leq\left(q_{1}+q_{2}\right)\left(q_{3}+q_{4}\right)$ and for any non-computable message $(z, m)$, $\operatorname{Pr}\left[f^{P, Q}(z, m)=c \mid \alpha_{P}, \alpha_{Q}\right] \leq \frac{1}{2^{n}-\max \left(q_{1}+q_{2}, q_{3}+q_{4}\right)}$. Moreover there is an efficient computable message extractor $\mathcal{E}_{\text {comp }}^{f}$ which can list all computable messages. 
Let $\mathbf{q}=\left(q_{1}, q_{2}, q_{3}, q_{4}\right)$. Now given the computable message extractor one can define a $\operatorname{PrA}$ extractor $\mathcal{E}^{f}$ as follows: $\mathcal{E}^{f}\left(y, \alpha_{P}, \alpha_{Q}\right)=(z, m)$ if there exists an unique computable message (from the list given by $\mathcal{E}_{\text {comp }}^{f}$ ) such that $f(z, m)=y$, otherwise it returns any arbitrary message.

Lemma 6. $\operatorname{Adv}_{H^{P, Q}}^{\mathrm{PI}}(\mathbf{q}, t) \leq \operatorname{Adv}_{f_{P, Q}}^{\mathrm{PI}}(\mathbf{q}, t) \leq \frac{\left(q_{1}+q_{2}\right)\left(q_{3}+q_{4}\right)}{2^{n}-\max \left(q_{1}+q_{2}, q_{3}+q_{4}\right)}$ for any $t$.

Lemma 7. Let $\mathbf{q}=\left(q_{1}, q_{2}, q_{3}, q_{4}\right)$ and let $f^{P, Q}=P(h \oplus m) \oplus Q(m) \oplus h$, where $P$ and $Q$ are invertible ideal permutations. For any preimage awareness (q, $e, t)$ adversary $A$ making at most $\mathbf{q}$ queries to the oracles $P, P^{-1}, Q, Q^{-1}$, there exists an extractor $\mathcal{E}$ such that

$\operatorname{Adv}_{f^{P, Q}, P, Q, \mathcal{E}}^{\mathrm{pra}}(A) \leq \frac{e\left(q_{1}+q_{2}\right)\left(q_{3}+q_{4}\right)}{2^{n}-\max \left(q_{1}+q_{2}, q_{3}+q_{4}\right)}+\frac{\left(q_{1}+q_{2}\right)^{2}\left(q_{3}+q_{4}\right)^{2}}{2\left(2^{n}-\max \left(q_{1}+q_{2}, q_{3}+q_{4}\right)\right)}$,

Theorem 2. Let $\operatorname{Gr} \varnothing \operatorname{stl} l^{\prime}(M)=P^{\prime}\left(H^{P, Q}(M)\right) \oplus H^{P, Q}(M)$ where $H^{P, Q}$ is Grøstl without the output transformation and $P, Q, P^{\prime}$ are independent random permutations. Then for any adversary making at most $q$ queries to all its oracles the PRO-advantage is bounded by $\ell_{\max } q^{2} q^{\prime 2} / 2^{n-2}$ if If $q^{\prime}=q_{1}+q_{2}+q_{3}+q_{4}+l_{\max } \leq$ $2^{n-1}$.

Proof. The result follows from Lemma 9, 10, 11 and 12, and Theorem 1

Remark 1. Our bound $\ell_{\max } q^{2} q^{\prime 2} / 2^{n-2}$ in the variant of Grøstl seems to be reasonable as we indeed have collision on the compression function in $2^{n / 4}$ complexity i.e. the collision advantage is $q^{4} / 2^{n}$. We believe the designers also noted that and this is why they consider at least double length hash function. We also strongly believe that the same bound can be achieved for original Grøstl. However to prove that we cannot apply Theorem 2 directly. This would be our one of the future research work.

\section{PRO Analysis of Hash Functions with PGV Output Transformations}

In the previous section, we considered the case that the finial output transformation is $O T(x)=E(x) \oplus x$. In this section, we consider 20 PGV compression functions shown in Table 1 as candidates of the final output transformation OT. Such $20 \mathrm{PGV}$ hash functions based on them were proved to be collision resistant in the ideal cipher model 4. More precisely, we will consider the case that $F^{P, E}(M)=O T\left(H^{P}\left(M_{1}\right), M_{2}\right)$, where $E$ is an ideal cipher, $M=M_{1} \| M_{2}$, $H^{P}\left(M_{1}\right)$ corresponds to $h_{i-1}$ and $M_{2}$ corresponds to $m_{i}$ in Table 1. Except for PGV 11, 13, 15-20 (See Example 2 and 3), Theorem 3 holds. The proof of the Theorem 3 is same as Davis-Meyer case. However we give the proof idea so that the reader could justify themselves. 
Proof Idea for 1-10, 12 and 14: Like to the Davis-Meyer case we only need to worry about the $E^{-1}$ query since we have chosen those PGV compression functions which behave like random oracle if adversary makes only $E$ queries. Note that 5-10 and 12 and $14 \mathrm{PGV}$ have $w_{i}$ as keys. So given a $E_{w}^{-1}(y)$ query simulator can make the list of all $h$ which can be computed, i.e. $H^{P}(M)=h$, and guess $m=h \oplus w$. Once simulator guesses $m$ he can make $\mathcal{F}$ queries $(M, m)$ and obtains responses $z$ 's. Now simulator can find a correct $m$ if $y$ is really obtained by some $\mathcal{F}(M, m)$. If there is no such $m$, simulator can response randomly and any bad behavior would be either bounded by the collision probability or by the preimage attack. The same argument works for PGV 1-4 since $H^{P}(M)$ is xor-ed with the the $E()$ output. So simulator can verify the correct $h$ among all computable hash outputs.

Example 2. See PGV 15 in Table1, which is $E_{m_{i}}\left(w_{i}\right) \oplus v$, where $w_{i}=h_{i-1} \oplus m_{i}$ and $v$ is a constant. Now we want to give an indifferentiable attack on $F^{P, E}$ based on PGV 15, even though $H^{P}$ is preimage aware, preimage resistant, and $\left(q, q_{H}, \epsilon\right)$-computable message aware with feasible $q$ and $q_{H}$ and negligible $\epsilon$. Let $H^{P}$ be an Merkle-damgård construction with strengthening, where the underlying compression function is a preimage aware function based on the ideal primitive $P$. As shown in 89, SMD (Merkle-damgård construction with strengthening) preserves preimage awareness of the compression function. Also SMD preserves preimage resistance. So, $H^{P}$ is also preimage aware and preimage resistant. We assume that $H^{P}$ is $\left(q, q_{H}, \epsilon\right)$-computable message aware with feasible $q$ and $q_{H}$ and negligible $\epsilon$. Now we construct an indifferentiability adversary $A$ for $F^{P, E}(M)=O T\left(H^{P}\left(M_{1}\right), M_{2}\right)$, where $O T(x, y)=E_{y}(x \oplus y) \oplus v$ is PGV 15 , and $v$ is a constant. First, $A$ chooses a random query $M=M_{1} \| M_{2}$ to $\mathcal{O}_{1}$, where $\left(\mathcal{O}_{1}, \mathcal{O}_{2}, \mathcal{O}_{3}, \mathcal{O}_{4}\right)$ is $\left(F^{P, E}, P, E, E^{-1}\right)$ or $\left(\mathcal{F}, S_{1}^{\mathcal{F}}, S_{2}^{\mathcal{F}}, S_{3}^{\mathcal{F}}\right)$ for any simulator $S^{\mathcal{F}}=\left(S_{1}^{\mathcal{F}}, S_{2}^{\mathcal{F}}, S_{3}^{\mathcal{F}}\right)$. $A$ gets its response $z$ from $\mathcal{O}_{1}$. And $A$ hands $\left(M_{2}, z \oplus v\right)$ over to $\mathcal{O}_{4}$. Then, $A$ gets its response $h$ from $\mathcal{O}_{4}$. $A$ makes a new query $\left(M_{2}^{\prime}, h \oplus M_{2} \oplus M_{2}^{\prime}\right)$ to $\mathcal{O}_{3}$. Then, $A$ gets its response $c$ from $\mathcal{O}_{3}$. Finally, $A$ hands $\left(M_{1} \| M_{2}^{\prime}\right)$ over to $\mathcal{O}_{1}$ and gets its response $z^{\prime}$. If $\left(\mathcal{O}_{1}, \mathcal{O}_{2}, \mathcal{O}_{3}, \mathcal{O}_{4}\right)$ is $\left(F^{P, E}, P, E, E^{-1}\right), c \oplus v=z^{\prime}$. On the other hand, since any simulator cannot know $M_{1}, c \oplus v \neq z^{\prime}$ with high probability. Therefore, $F^{P, E}$ based on PGV 15 is not indifferentiable from a VIL random oracle $\mathcal{F}$. In the similar way, cases of PGV 11, 13, and 16 are not secure.

Example 3. See PGV 17 in Table 1, which is $E_{h_{i-1}}\left(m_{i}\right) \oplus m_{i}$. Firstly, we define a hash function $H^{P}(x):\{0,1\}^{*} \rightarrow\{0,1\}^{n}$ as follows, where $c$ is any $n$-bit constant and $P$ is a VIL random oracle with $n$-bit output size.

$$
H^{P}(x)= \begin{cases}c, & \text { if } x=c ; \\ P(x), & \text { otherwise }\end{cases}
$$

In the similar way with the proofs of Section 6 , we can prove that $H^{P}$ is preimage aware, preimage resistant, $\left(q, q_{H}(=q+1), 1 / 2^{n}\right)$-computable message 
aware, where $q_{H}$ is the number of computable messages obtained from $q$ inputoutput pairs of $P$. Now we want to give an indifferentiable attack on $F^{P, E}$ based on PGV 17. We construct an indifferentiability adversary $A$ for $F^{P, E}(M)=$ $O T\left(H^{P}\left(M_{1}\right), M_{2}\right)$, where $O T(x, y)=E_{x}(y) \oplus y$ is PGV 17. First, $A$ chooses a query $M=c \| M_{2}$ to $\mathcal{O}_{1}$, where $M_{2}$ is a randomly chosen one-block message. $A$ gets its response $z$ from $\mathcal{O}_{1}$. And $A$ hands $\left(c, z \oplus M_{2}\right)$ over to $\mathcal{O}_{4}$. Then, $A$ gets its response $m$ from $\mathcal{O}_{4}$. If $\left(\mathcal{O}_{1}, \mathcal{O}_{2}, \mathcal{O}_{3}, \mathcal{O}_{4}\right)$ is $\left(F^{P, E}, P, E, E^{-1}\right)$, $m=M_{2}$. On the other hand, since any simulator cannot know $M_{2}, m \neq M_{2}$ with high probability. Therefore, $F^{P, E}$ based on PGV 17 is not indifferentiable from a VIL random oracle $\mathcal{F}$. In the similar way, cases of PGV 18-20 are not secure.

Theorem 3. [PRO Construction via 12 PGVs]

Let $F^{P, E}(M)=O T\left(H^{P}\left(M_{1}\right), M_{2}\right)$, where $M=M_{1} \| M_{2}$, OT is any $P G V$ constructions except for $P G V$ 11, 13, 15-20, $E^{-1}$ is efficiently computable, and $E$ is an ideal cipher. For any indifferentiability adversary $A$ making at most $\left(q_{0}, q_{1}, q_{2}, q_{3}\right)$ queries to its four oracles with bit-size $l_{\text {max }}$ for the longest $\mathcal{O}_{0}$ query, there exists a $\operatorname{Pr} A\left(q, q_{2}+1, t\right)$-adversary $C_{A}$ with runtime $t=\operatorname{Time}(A)+$ $O\left(q_{2} \cdot \operatorname{Time}(\mathcal{E})+q_{0}+q_{1}+\left(q_{2}+q_{0}\right) N Q\left[l_{\text {max }}\right]\right)$ and

$$
\begin{gathered}
\mathbf{A d v}_{F, S}^{\mathrm{pro}}(A) \leq \mathbf{A} \mathbf{d} \mathbf{v}_{H^{P}, P, \mathcal{E}}^{\mathrm{pra}}\left(C_{A}\right)+q_{3} \times \mathbf{A d}_{\mathbf{d}_{H^{P}}^{\mathrm{PI}}}^{\mathrm{PI}}(q, t) \\
\quad+q_{0} q_{3} \epsilon+\frac{2 q_{0} q_{3}+q_{2} q_{0}}{2^{n}-q_{0}-q_{2}-q_{3}}+\frac{\left(q_{H}+q_{2}+q_{0}\right)^{2}}{2^{n+1}},
\end{gathered}
$$

where $H^{P}(\cdot)$ is preimage resistant and $\left(q^{*}, q_{H}, \epsilon\right)$-computable message aware for an efficient computable message extractor $\mathcal{E}_{\mathrm{comp}}$ where $q^{*}=q_{1}+q_{2} N Q\left[l_{\max }\right]$.

Proof. The proof is very similar to that of Theorem [1 It will be referred to the full paper [5].

Table 1. 20 Collision Resistant PGV Hash Functions in the Ideal Cipher Model 44. $\left(w_{i}=m_{i} \oplus h_{i-1}\right)$

\begin{tabular}{|c|c||c|c|}
\hline Case & PGV & Case & PGV \\
\hline 1 & $E_{m_{i}}\left(h_{i-1}\right) \oplus h_{i-1}$ & 11 & $E_{m_{i}}\left(h_{i-1}\right) \oplus v$ \\
\hline 2 & $E_{m_{i}}\left(w_{i}\right) \oplus w_{i}$ & 12 & $E_{w_{i}}\left(h_{i-1}\right) \oplus v$ \\
\hline 3 & $E_{m_{i}}\left(h_{i-1}\right) \oplus w_{i}$ & 13 & $E_{m_{i}}\left(h_{i-1}\right) \oplus m_{i}$ \\
\hline 4 & $E_{m_{i}}\left(w_{i}\right) \oplus h_{i-1}$ & 14 & $E_{w_{i}}\left(h_{i-1}\right) \oplus w_{i}$ \\
\hline 5 & $E_{w_{i}}\left(m_{i}\right) \oplus m_{i}$ & 15 & $E_{m_{i}}\left(w_{i}\right) \oplus v$ \\
\hline 6 & $E_{w_{i}}\left(h_{i-1}\right) \oplus h_{i-1}$ & 16 & $E_{m_{i}}\left(w_{i}\right) \oplus m_{i}$ \\
\hline 7 & $E_{w_{i}}\left(m_{i}\right) \oplus h_{i-1}$ & 17 & $E_{h_{i-1}}\left(m_{i}\right) \oplus m_{i}$ \\
\hline 8 & $E_{w_{i}}\left(h_{i-1}\right) \oplus m_{i}$ & 18 & $E_{h_{i-1}}\left(w_{i}\right) \oplus w_{i}$ \\
\hline 9 & $E_{w_{i}}\left(m_{i}\right) \oplus v$ & 19 & $E_{h_{i-1}}\left(m_{i}\right) \oplus w_{i}$ \\
\hline 10 & $E_{w_{i}}\left(m_{i}\right) \oplus w_{i}$ & 20 & $E_{h_{i-1}}\left(w_{i}\right) \oplus m_{i}$ \\
\hline
\end{tabular}




\section{PRO Attacks on Hash Functions with Some DBL Output Transformations}

Here, we consider DBL (Double Block Length) output transformations. Unfortunately, many constructions with DBL output transformations are not indifferentiability secure, even though $H^{P}$ satisfies all requirements as mentioned before.
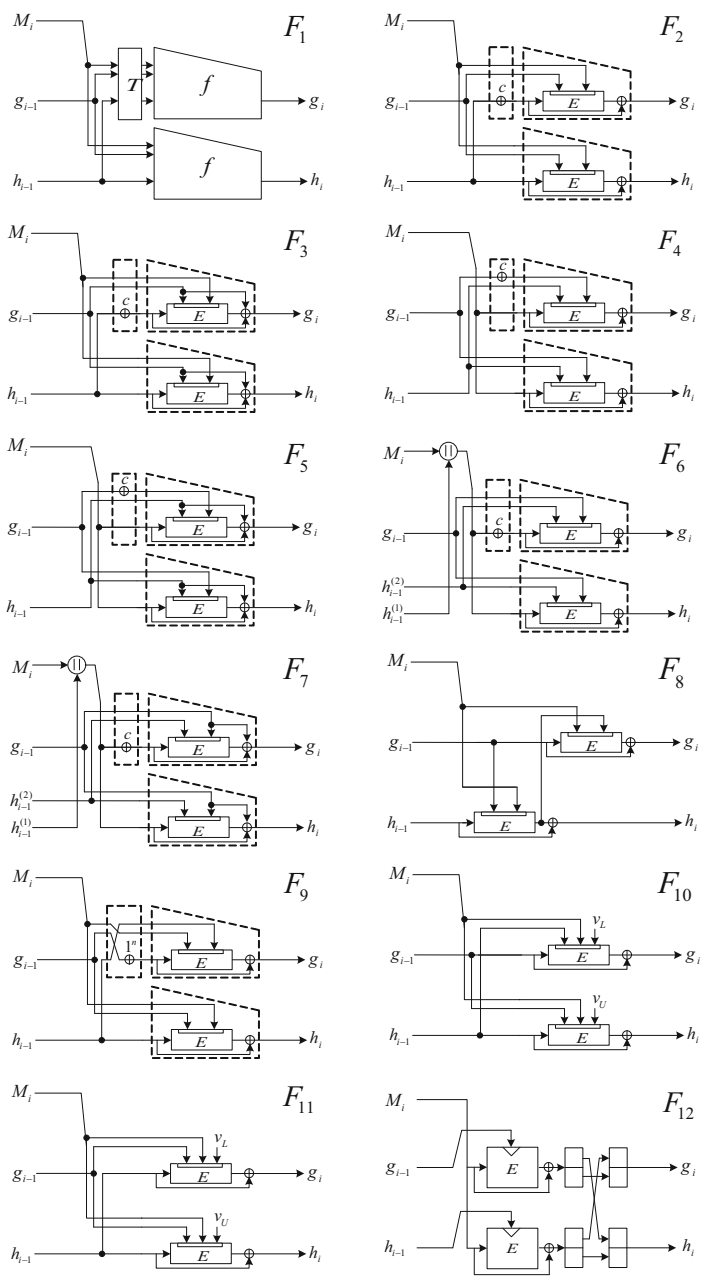

Fig. 3. Double Block Length constructions : $F_{i} i=1$ to 12 


\subsection{The Case of $O T(x)=f(x) \| f(x \oplus p)$}

There are several DBL compression functions of the form $O T(x)=f(x) \| f(x \oplus p)$ [19]13] where $p$ is a non-zero constant and $f$ is any function. See Fig. 3, where $F_{1}$ was proposed by Nandi in [19] and $F_{2} \sim F_{7}$ were proposed by Hirose in [13]. In fact, the $T$ in $F_{1}$ Fig. [3 is a permutation without any fixed point and $T^{2}=i d$. Here, we consider only $T(x)=x \oplus p$, where $p$ is a non-zero constant. We define a hash function $H^{P}(x):\{0,1\}^{*} \rightarrow\{0,1\}^{n}$ as follows, where $c$ is any $n$-bit constant and $P$ is a VIL random oracle with $n$-bit output size. $1^{n}$ and $0^{n}$ indicate the $n$-bit one and zero strings.

$$
H^{P}(x)= \begin{cases}c \oplus p, & \text { if } x=0^{n} \\ c, & \text { if } x=1^{n} \\ P(x), & \text { otherwise }\end{cases}
$$

The following theorems show that $H^{P}$ is preimage aware, $q_{H}$ is small (more precisely computable-awareness) and preimage resistant. The proofs are given in the full version [5].

Theorem 4. Let $H^{P}$ be the above hash function. For any preimage awareness $(q, e, t)$-adversary $A$ making at most $q$ queries to the oracles $P$, there exists an extractor $\mathcal{E}$ such that

$$
\operatorname{Adv}_{H^{P}, P, \mathcal{E}}^{\mathrm{pra}}(A) \leq \frac{e q}{2^{n}}+\frac{(q+2)^{2}}{2^{n+1}}, \text { and } q_{H} \leq q+2 .
$$

Theorem 5. Let $H^{P}$ be the above hash function. Let $q$ be the maximum number of queries to $P$. For any preimage-finding adversary $A$ with $q$ queries to $P$, $\operatorname{Adv}_{H^{P}}^{\mathrm{PI}}(A) \leq \frac{3+q}{2^{n}}$. For any $n$-bit $y$ and any $M$ not computable from any advise string $\alpha$ which consists of $q$ query-response pairs of $P, \operatorname{Pr}\left[H^{P}(M)=y \mid \alpha\right] \leq 1 / 2^{n}$.

Indifferentiability Attack on $F(M)=H^{P}(M) \|\left(H^{P}(M) \oplus p\right)$.

Let $\left(\mathcal{O}_{1}, \mathcal{O}_{2}, \mathcal{O}_{3}\right)$ be $\left(F^{P, f}, P, f\right)$ or $\left(\mathcal{F}, S_{1}^{\mathcal{F}}, S_{1}^{\mathcal{F}}\right)$ for any simulator $S$. Now we define an adversary $A$ as follows. First, $A$ makes query ' 0 ' and ' 1 ' to $\mathcal{O}_{1}$. Then, $A$ obtains responses $\left(a_{1} \| a_{2}\right)$ and $\left(b_{1} \| b_{2}\right)$. If $\mathcal{O}_{1}=F$, then $a_{1}=b_{2}$ and $a_{2}=b_{1}$. But, if $\mathcal{O}_{1}=\mathcal{F}, a_{1}=b_{2}$ and $a_{2}=b_{1}$ with the probability $1 / 2^{n}$. So, $F$ is not indifferentiably secure.

\subsection{PRO Attack with $O T(x)=F_{i}(x)$ for $i=8,12$, (Fig. 3)}

In the case of $F_{8}$ proposed by Lai and Massey in [15, which is called Tandem DM, there is the following structural weakness. If for any $a g_{i-1}=h_{i-1}=M_{i}=a$ in $F_{8}$ of Fig. 3, then $h_{i} \oplus g_{i}=a$. We can show an indifferentiability attack on $F(M)=F_{9}\left(H^{P}(M)\right)$, where $H^{P}$ is preimage aware and $q_{H}$ is small. We define a hash function $H^{P}(x):\{0,1\}^{*} \rightarrow\{0,1\}^{n}$ as follows, where $c$ is any $n / 2$-bit constant and $P$ is a VIL random oracle with $n$-bit output size.

$$
H^{P}(x)= \begin{cases}c \| c, & \text { if } x=0 \\ P(x), & \text { otherwise }\end{cases}
$$


We can easily show that $H^{P}$ is preimage aware, preimage resistant and $\left(q_{P}, q_{H}(=\right.$ $\left.q_{P}+1\right), 1 / 2^{n}$-computable message aware, where $q_{H}$ is the number of computable messages obtained from $q_{P}$ input-output pairs of $P$. and $q_{H}$ is small.

Then, we show that $F\left(M_{1} \| M_{2}\right)=F_{8}\left(H^{Q}\left(M_{1}\right), M_{2}\right)$ is not indifferentiable from a VIL random oracle $\mathcal{F}$ as follows. $A$ makes a query ' $(0 \| c)^{\prime}$ ' to $\mathcal{O}_{1}$ and get its response $z=\left(z_{1} \| z_{2}\right)$. $A$ checks if $z_{1} \oplus z_{2}=c$. If $\mathcal{O}_{1}$ is $\mathcal{F}, z_{1} \oplus z_{2}=c$ with the probability $1 / 2^{n / 2}$. On the other hand, if $\mathcal{O}_{1}$ is $F, z_{1} \oplus z_{2}=c$ with probability 1 . So $F$ is not indifferentiability secure. In the case of $F_{12}$, which is called MDC-2, if the values of $M_{i}$ and $h_{i-1}$ are fixed, the half of bits of the output of $F_{12}$ is also fixed regardless of what $g_{i-1}$ is. Using this weakness, in a similar way as shown in above, we also can construct $H^{P}$ such that $F\left(M_{1} \| M_{2}\right)=F_{12}\left(H^{P}\left(M_{1}\right), M_{2}\right)$ is not indifferentiably secure.

\section{Conclusion}

In this paper we extend the applicability of preimage-awareness in those hash functions whose output transformation cannot be modeled as a random oracle. We choose Davis-Meyer as an output transformation based on a random permutation and show that the hash function is PRO if $H^{P}$ is PrA, preimage resistant and computable message aware. The computable message awareness is a new notion introduced here similar to PrA. However this is not same as $\operatorname{PrA}$ as we can see the separation among these notions. As an application to our result we prove the PRO property of a variant of Grøstl hash function. We similarly prove that 12 PGV compression function out of 20 collision resistant PGV hash functions can be employed as output transformation with the similar assumption on $H^{P}$. However, some the popular double length hash function can not be used as we have shown PRO attacks. In summary, we study the choice of output transformation beyond the random oracle model and found both positive and negative results.

\section{References}

1. Andreeva, E., Mennink, B., Preneel, B.: On the Indifferentiability of the Grøstl Hash Function. In: Garay, J.A., De Prisco, R. (eds.) SCN 2010. LNCS, vol. 6280, pp. 88-105. Springer, Heidelberg (2010)

2. Bellare, M., Kohno, T., Lucks, S., Ferguson, N., Schneier, B., Whiting, D., Callas, J., Walker, J.: Provable Security Support for the Skein Hash Family, http://www.skein-hash.info/sites/default/files/skein-proofs.pdf

3. Bellare, M., Rogaway, P.: The Security of Triple Encryption and a Framework for Code-Based Game-Playing Proofs. In: Vaudenay, S. (ed.) EUROCRYPT 2006. LNCS, vol. 4004, pp. 409-426. Springer, Heidelberg (2006)

4. Black, J.A., Rogaway, P., Shrimpton, T.: Black-box analysis of the block-cipherbased hash-function constructions from PGV. In: Yung, M. (ed.) CRYPTO 2002. LNCS, vol. 2442, pp. 320-335. Springer, Heidelberg (2002) 
5. Chang, D., Nandi, M., Yung, M.: On the Security of Hash Functions Employing Blockcipher Postprocessing, http://eprint.iacr.org/2010/629

6. Coron, J.S., Dodis, Y., Malinaud, C., Puniya, P.: Merkle-Damgard Revisited: How to Construct a Hash Function. In: Shoup, V. (ed.) CRYPTO 2005. LNCS, vol. 3621, pp. 430-448. Springer, Heidelberg (2005)

7. Damgård, I.B.: A design principle for hash functions. In: Brassard, G. (ed.) CRYPTO 1989. LNCS, vol. 435, pp. 416-427. Springer, Heidelberg (1990)

8. Dodis, Y., Ristenpart, T., Shrimpton, T.: Salvaging Merkle-Damgård for Practical Applications. In: Joux, A. (ed.) EUROCRYPT 2009. LNCS, vol. 5479, pp. 371-388. Springer, Heidelberg (2009)

9. Dodis, Y., Ristenpart, T., Shrimpton, T.: Salvaging Merkle-Damgård for Practical Applications. Full version of [6], Cryptology ePrint Archive: Report 2009/177

10. Ferguson, N., Lucks, S., Schneier, B., Whiting, D., Bellare, M., Kohno, T., Callas, J., Walker, J.: The Skein Hash Function Family. Submission to NIST (2008)

11. Gauravaram, P., Knudsen, L.R., Matusiewicz, K., Mendel, F., Rechberger, C., Schläffer, M., Thomsen, S.S.: Grøstl - a SHA-3 candidate. Submission to NIST (2008)

12. Hirose, S.: Secure Double-Block-Length Hash Functions in a Black-Box Model. In: Park, C.-s., Chee, S. (eds.) ICISC 2004. LNCS, vol. 3506, pp. 330-342. Springer, Heidelberg (2005)

13. Hirose, S.: How to Construct Double-Block-Length Hash Functions. In: Second Hash Workshop (2006)

14. Kelsey, J.: Some notes on Grøstl (2009), http://ehash.iaik.tugraz.at/uploads/d/d0/Grostl-comment-april28.pdf

15. Lai, X., Massey, J.L.: Hash Functions Based on Block Ciphers. In: Rueppel, R.A. (ed.) EUROCRYPT 1992. LNCS, vol. 658, pp. 55-70. Springer, Heidelberg (1993)

16. Maurer, U.: Indistinguishability of Random Systems. In: Knudsen, L.R. (ed.) EUROCRYPT 2002. LNCS, vol. 2332, pp. 110-132. Springer, Heidelberg (2002)

17. Maurer, U., Renner, R., Holenstein, C.: Indifferentiability, Impossibility Results on Reductions, and Applications to the Random Oracle Methodology. In: Naor, M. (ed.) TCC 2004. LNCS, vol. 2951, pp. 21-39. Springer, Heidelberg (2004)

18. Merkle, R.C.: One way hash functions and DES. In: Brassard, G. (ed.) CRYPTO 1989. LNCS, vol. 435, pp. 428-446. Springer, Heidelberg (1990)

19. Nandi, M.: Towards Optimal Double-Length Hash Functions. In: Maitra, S., Veni Madhavan, C.E., Venkatesan, R. (eds.) INDOCRYPT 2005. LNCS, vol. 3797, pp. 77-89. Springer, Heidelberg (2005)

20. Preneel, B., Govaerts, R., Vandewalle, J.: Hash Functions Based on Block Ciphers: A Synthetic Approach. In: Stinson, D.R. (ed.) CRYPTO 1993. LNCS, vol. 773, pp. 368-378. Springer, Heidelberg (1994)

21. Winternitz, R.: A Secure Hash Function built from DES. In: Proceedings of the IEEE Symp. on Information Security and Privacy, pp. 88-90. IEEE Press, Los Alamitos (1984)

\section{Appendix A. Revisiting the Proof of $" \operatorname{RO}(\operatorname{PrA}(\cdot))=\operatorname{PRO}(\cdot) "$}

In [8]9] it was proved that $F^{R, P}(M)=\mathcal{R}\left(H^{P}(M)\right)$ is indifferentiable from a VIL random oracle $\mathcal{F}$, where $\mathcal{R}:\{0,1\}^{m} \rightarrow\{0,1\}^{n}$ is a FIL random oracle, $P$ is an ideal primitive, and $H^{P}: \mathcal{M} \rightarrow\{0,1\}^{m}$ is preimage-aware. The result can 
be used to prove the indifferentiable security of any hash function which uses a post-processor defined independently from the underlying iteration function $P$. In the course of our studies we have found that the proof given in [89] is not completely correct (though the claims remain correct). We have reported this in a limited distribution abstracts on February and May 2010 (recently in October 2010, a correction on the e-print version has appeared by the original coauthors, further confirming our findings). Let us review the issues. There are two main flaws (to be described below) in the proof, and we need to provide alternative definitions of simulator and preimage-aware attacker to fix them. (We note that while somewhat technical, the revision is crucial). Let NQ $[l]$ be the number of $P$ queries required for the computation of $H^{P}(M)$ for $|M|=l$. We denote Time $(\cdot)$ and STime $(\cdot)$ to mean the run time and simulation run time of an algorithm. Now we restate the Theorem 4.1. in 89] (in terms of our PrA terminologies) and provide a sketch of the proof given in [8].

\section{Theorem 4.1 of 89}

For any given efficient extractor $\mathcal{E}$, there exists a simulator $S=\left(S_{1}, S_{2}\right)$ with Time $(S)=O\left(q_{1} \cdot \operatorname{STime}(P)+q_{2} \cdot \operatorname{Time}(\mathcal{E})\right)$. The simulator makes at most $q_{2} \mathcal{F}$-queries. For any indifferentiability adversary $A^{\mathcal{O}_{0}, \mathcal{O}_{1}, \mathcal{O}_{2}}$ making at most $\left(q_{0}, q_{1}, q_{2}\right)$ queries to its three oracles with bit-size $l_{\max }$ for the longest $\mathcal{O}_{0^{-}}$ query, there exists a $\left(q_{1}+q_{0} \cdot \mathrm{NQ}\left[l_{\max }\right], q_{2}+1, t\right)$-PrA adversary $B_{A}^{P}$ with runtime $t=\operatorname{Time}(A)+O\left(q_{0} \cdot \mathrm{NQ}\left[l_{\text {max }}\right]+q_{1}+q_{2} \operatorname{Time}(\mathcal{E})\right)$ such that

$$
\mathbf{A d v}_{F, S}^{\text {pro }}(A) \leq \mathbf{A d d v} H_{H^{P}, P, \mathcal{E}}^{\text {pra }}\left(B_{A}\right) .
$$

Outline of Proof of Theorem 4.1. of [9]. Let $\mathcal{E}$ be an arbitrary extractor for $H$. Then $S=\left(S_{1} ; S_{2}\right)$ works as follows. It maintains an internal advice string $\alpha$ (initially empty) that will consist of pairs $(u ; v)$ corresponding to $A$ 's queries to $P$ (via $S_{1}$ ). When $A$ queries $u$ to $S_{1}$, the simulator simulates $v \leftarrow$ $P(u)$ appropriately, sets $\alpha \leftarrow \alpha \|(u ; v)$, and returns $v$. For a query $Y$ to $S_{2}$, the simulator computes $X \leftarrow \mathcal{E}(Y ; \alpha)$. If $X=\perp$ then the simulator returns a random point. Otherwise it simulates $Z \leftarrow \mathcal{F}(X)$ and returns $Z$ to the adversary. The games $R 0, I 1, G 0, G 1$ and $B_{A}$ have been defined in [9] and the authors claimed the following:

$$
\text { (1) } G 1 \equiv I 1 \equiv\left(\mathcal{F}, S_{1}, S_{2}\right), \quad(2) \quad G 0 \equiv R 0 \equiv\left(F^{P, \mathcal{R}}, P, \mathcal{R}\right) .
$$

Due to the above claim the PRO-advantage of any adversary $A$ is nothing but $\mid \operatorname{Pr}\left[A^{G 0}=1\right]-\operatorname{Pr}\left[A^{G 1}=1\right]$. From the pseudocodes of games $G 0$ and $G 1$, it is easy to see that they are identical-until-Bad. Hence $\operatorname{Addv}_{F, S}^{\text {pro }}(A) \leq$ $\operatorname{Pr}\left[A^{G 1}\right.$ sets Bad true]. The proof proceeds by defining a PRA-adversary $B_{A}$ which makes preimage-aware attack successfully whenever $B_{A}$ sets Bad true. Since $B_{A}$ sets Bad true only if it finds a collision of $H^{P}$ or finds a message $M$ such that $\mathcal{E}(\alpha, Y) \neq M$ where $Y=H^{P}(M)$. So $\operatorname{Pr}\left[B_{A}\right.$ sets Bad true $] \leq$ $\mathbf{A d v}_{H^{P}, P, \mathcal{E}}^{\text {pra }}\left(B_{A}\right)$. The theorem follows immediately from the following claim:

(3) $\operatorname{Pr}\left[A^{G 1}\right.$ sets Bad true $] \leq \operatorname{Pr}\left[B_{A}\right.$ sets Bad true $]$. 


\subsection{Problems in Proof of Theorem 4.1. of [9]}

In this section we explain the flaws we observe in the proof of Theorem 4.1. of [9. To understand it one needs to go through the definitions of the games $G 0$, $R 0, G 1$ and $G(B)$ (the tuple of three oracles simulated by $B$ ) described in [9] 2 ,

Flaw 1. $G 0$ is not equivalent to $R 0$.

If $\mathcal{O}_{0}$ in $G 0$ has not been queried before (so the Bad event in $G 0$ would not occur) then the output of $\mathcal{O}_{2}(Y)$ query is $\mathrm{F}(X)$ whenever $X=\mathcal{E}(Y, \alpha) \neq \perp$, otherwise it returns $\mathrm{R}(X)$ where $\mathrm{F}$ and $\mathrm{R}$ perfectly simulate two independent random oracles $\mathcal{F}$ and $\mathcal{R}$ respectively. We show that $\mathcal{O}_{2}$ cannot be equivalent to a random oracle. Suppose $\mathcal{E}$ is an extractor which returns a special message $M^{*}$ whenever the advise string $\alpha$ is empty. If $A$ makes first two successive distinct $\mathcal{O}_{2}$ queries $Y_{2,1}$ and $Y_{2,2}$ then $X_{2,1}=X_{2,2}=M^{*}$ and hence the outputs of $\mathcal{O}_{2}$ in game $G 0$ are identical (same as $\mathrm{F}\left[M^{*}\right]$ ).

To get rid of the above problem, we can do the following steps in $\mathcal{O}_{2}$ (also in simulator $S_{2}$ ) immediately after it obtains $X=\mathcal{E}(Y, \alpha)$ : Compute $H^{P}(X)=Y^{\prime}$ and check whether it is the same as $Y$ or not. If extractor returns a correct message, i.e. $Y=Y^{\prime}$, then $S_{2}$ or $\mathcal{O}_{2}$ returns $\mathcal{F}(M)$. Otherwise, it returns randomly. To compute $H^{P}(X)$ one might need to simulate some $P$ outputs (in case of $G 0$ ) or make $P$-queries (in case of $\mathcal{O}_{2}$ of $B$ ).

\section{Flaw 2. $G(B) \not \equiv G 1$ and $G 1, G(B)$ are not identical-until-Bad.}

We first observe that the advise string $\alpha$ in $G 1$ is not the same as that of $B$ since the advice string $\alpha$ is updated whenever $A$ has access to the oracle $\mathcal{O}_{1}$ in Game $G 1$, but the advice string is updated whenever $A$ has access to the oracle $\mathcal{O}_{0}$ and $\mathcal{O}_{1}$ of $B$ in Fig 3 of [9]. For example, let $\mathcal{E}(Y, \alpha)$ return a message $M$ whenever $H^{P}(M)=Y$ is "computable' from $\alpha$ otherwise return $\perp$. Any adversary which can guess $H^{P}(M)$ correctly and turn it to $\mathcal{O}_{2}$ query then $\mathcal{O}_{2}^{B}(Y \mid \tau)$ returns $z$. However, $\mathcal{O}_{2}^{G 1}(Y \mid \tau)$ returns a random string $\mathrm{R}[Y]$ since $\alpha$ is the empty string in $A^{G 1}$. So $G(B) \not \equiv G 1$. One can similarly show that $G 1, G(B)$ are not identicaluntil-Bad.

A possible attempt is to update the advise string for $\mathcal{O}_{0}$ queries in all games, in particular $G 1$. However, if we do so then the simulator is not independent of $\mathcal{F}$ queries (since the advise string is updated whenever there is a $\mathcal{O}_{0}$-query and the advise string is used to define the response of $S_{2}$ ). On the other hand, we cannot ignore the $H^{P}(M)$ computation in $B$ for $\mathcal{O}_{0}$ queries of $A$. This computation is essential to making $\operatorname{PrA}$ attack successfully. It seems impossible to handle the advise string so that it is updated in the same way for all games as well as $H^{P}(\cdot)$ computations are made for $\mathcal{O}_{0}$-queries. We can solve the problem if we postpone the computation of $H^{P}$ until all queries of $A$ are made. So we need a finalization procedure in $B$ which essentially does all $H^{P}(M)$ computations of $\mathcal{O}_{0}(M)$-queries.

${ }^{2}$ We have defined the revised version of these games in the paper. We refer readers to 9] to see the original definitions to understand the flaws. 


\subsection{Revised Proof of Theorem 4.1 of [9]}

We state the corrected version of theorem 4.1. below. The revised version of $B:=$ $B_{A}$, simulators and the games $G 0$ and $G 1$ are defined in Fig. 1. The adversary $B_{A}$ has a subroutine called Finish() which is defined trivially. It mainly completes the PrA attack. It is easy to see that whenever Finish() is being executed either we have a collision in $H^{P}$ or there is some message $M$ such that $H^{P}(M)=$ $y,(y, M) \notin$ Ext. For simplicity we ignore the details of the subroutine. Let $q=$ $q_{1}+\left(q_{0}+q_{2}\right) \cdot \mathrm{NQ}\left[l_{\max }\right]$.

Lemma 8. $G 1 \equiv\left(\mathcal{O}_{0}^{B_{A}}, \mathcal{O}_{1}^{B_{A}}, \mathcal{O}_{2}^{B_{A}}\right) \equiv\left(\mathcal{F}, S_{1}, S_{2}\right)$. Games $G 0$ and $G 1$ are identical-until-Bad.

The Lemma is obvious from the games described in Fig. 1. We leave readers to verify. The following lemma essentially says that $G 0$ is equivalent to $\left(\mathcal{R}\left(H^{P}\right), P, \mathcal{R}\right)$. The proof of the lemma is easy to verify and we skip it for the full version.

Lemma 9. $G 0 \equiv\left(\mathcal{R}\left(H^{P}\right), P, \mathcal{R}\right)$, i.e. for any distinguisher $A$, the output distribution of $A^{G 0}$ and $A^{\mathcal{R}\left(H^{P}\right), P, \mathcal{R}}$ are identically distributed.

Lemma 10. Whenever $A^{G 1}$ sets Bad true, $B_{A}$ sets Bad true and $B_{A}$ makes $\operatorname{Pr} A$ attack successful. So we have $\operatorname{Pr}\left[A^{G 1}\right.$ sets bad $] \leq \operatorname{Pr}\left[B_{A}\right.$ sets bad $] \leq$ $\mathbf{A d v}_{H^{P}, P, \mathcal{E}}^{\text {pra }}\left(B_{A}\right)$.

Proof. We already know from Lemma 8 that $G 1$ is equivalent to the oracles simulated by $B_{A}$. However, the two games defined bad event in different manners. The game $G 1$ sets bad during the computation of responses whereas the adversary $B_{A}$ sets bad after all responses of the queries. $A^{G 1}$ sets bad true in line 209, 003 and in line 206, 005. We can see that if the conditional statements written in 209 and 003 in game $G 1$ hold then we have a collision in $H^{P}$ (there exist $M \neq X$ such that $\left.H^{P}(M)=H^{P}(X)\right)$. So we have PrA attack which is taken care of in 401 in the second step of $B_{A}$. For the lines 205 and 005 we have $M$ such that $H^{P}(M)=y$ and $\operatorname{Ext}[y] \neq M$, i which case PrA attack is possible due to incorrect guess of the extractor. This has been taken care of in 403 .

By using the above lemmas the theorem follows immediately

Theorem 6 (Ro domain extension via $\operatorname{Pr} \mathbf{A})$. For any given extractor $\mathcal{E}$ we can construct a simulator $S=\left(S_{1}, S_{2}\right)$ with Time $(S)=O\left(\left(q_{1}+q_{2} \cdot N Q\left[l_{\text {max }}\right]\right)\right.$. STime $\left.(P)+q_{2} \cdot \operatorname{Time}(\mathcal{E})\right)$. For any indifferentiability adversary $A^{\mathcal{O}_{0}, \mathcal{O}_{1}, \mathcal{O}_{2}}$ making at most $\left(q_{0}, q_{1}, q_{2}\right)$ queries to its three oracles with bit-size $l_{\text {max }}$ for the longest $\mathcal{O}_{0}$-query, there exists a $\left(q, q_{2}+1, t\right)$-adversary $B$ with runtime $t=\operatorname{Time}(A)+$ $O\left(q_{2} \cdot \operatorname{Time}(\mathcal{E})+q_{0}+q_{1}+\left(q_{2}+q_{0}\right) N Q\left[l_{\text {max }}\right]\right)$ and

$$
\mathbf{A d v}_{F, S}^{\text {pro }}(A) \leq \mathbf{A d v}_{H^{P}, P, \mathcal{E}}^{\mathrm{pra}}(B),
$$




\begin{tabular}{|c|c|}
\hline \begin{tabular}{|l|l|l} 
Game & $G 0$ & and $G 1$ \\
\cline { 2 - 2 }
\end{tabular} & 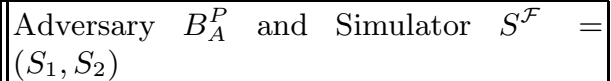 \\
\hline $\begin{array}{l}\text { Initialize }: \mathrm{H}=\mathrm{R}_{2}=\mathrm{R}_{0}=\phi ; \\
\mathcal{L}=\beta=\phi, i=1, \mathrm{Bad}=\mathrm{F}\end{array}$ & $\begin{array}{l}\text { Initialize }: \mathrm{H}=\mathrm{R}_{2}=\mathrm{R}_{0}=\mathcal{L}=\beta=\phi ; \\
i=1, \mathrm{Bad}=\mathrm{F} ; \\
\text { Run } A \text { and respond queries of } A \text { 's as fol- } \\
\text { lows: }\end{array}$ \\
\hline 200 On $\mathcal{O}_{2}-$ query $y:=y$ & 200 On $\mathcal{O}_{2}\left(\right.$ or $\left.S_{2}\right)$-query $y:=y_{i}, i=i+1$ \\
\hline $1 \quad X=\mathcal{E}\left(y_{i}, \beta\right) ;$ Ext & 201 $X=\mathcal{E}\left(y_{i}, \beta\right)$; Ext $\stackrel{\cup}{\longleftarrow}(y, X)$; \\
\hline$y^{\prime}=H^{P}(X)$ and update $\beta$ & 202 $y^{\prime}=H^{P}(X)$ and update $\beta$; \\
\hline If $y^{\prime} \neq y$ & If $y^{\prime} \neq y$ \\
\hline then $z=\mathcal{R}(y)$ & then $z=\mathcal{R}(y)$ \\
\hline If $y^{\prime} \neq y \wedge(M, y) \in \mathrm{H}$ & \\
\hline then $\operatorname{Bad}=\mathrm{T} ; z=\mathrm{R}_{0}[y]$ & \\
\hline $\begin{array}{l}\text { If } y^{\prime}=y \\
\text { then } z=\mathcal{F}(X\end{array}$ & $\mid \begin{array}{ll}207 & \text { If } y^{\prime}=y \\
208 & \text { then } z=\mathcal{F}(X)\end{array}$ \\
\hline $\begin{array}{l}\text { then } z=\mathcal{F}(X) \\
\text { If } y^{\prime}=y \wedge(M, y) \in \mathrm{H} \wedge M \neq X\end{array}$ & then $z=\mathcal{F}(X)$; \\
\hline then $\operatorname{Bad}=\mathrm{T} ; z=\mathrm{R}_{0}[y] ;$ & \\
\hline $\mathrm{R}_{2} \longleftarrow(y, z) ;$ return $z ;$ & |211 $\quad \mathrm{R}_{2} \stackrel{\cup}{\longleftarrow}(y, z)$; return $z$; \\
\hline 100 On $\mathcal{O}_{1}-$ query $u$ & 100 On $\mathcal{O}_{1}\left(\right.$ or $\left.S_{1}\right)$-query $u$ \\
\hline$v=P(u$ & $\| 101 v=P(u) ; \beta \stackrel{\|}{\longleftarrow}(u, v)$; \\
\hline return $v$ & return $v$; \\
\hline 000 On $\mathcal{O}_{0}$ - query $M$ & |000 On $\mathcal{O}_{0}($ or $\mathcal{F})$ - query $M$ \\
\hline$z=\mathcal{F}(M$ & $z=\mathcal{F}(M) ; \mathcal{L} \longleftarrow M ;$ \\
\hline$y=H^{P}(M) ; \mathrm{H} \longleftarrow(M, y) ;$ & \|002 $\quad \mathrm{R}_{0} \longleftarrow(y, z)$; return $z$; \\
\hline If $\mathrm{R}_{0}[y] \neq \perp$ & $\begin{array}{l}400 \text { Finalization: (after } A \text { finishes } \\
\text { queries.) }\end{array}$ \\
\hline then $\operatorname{Bad}=\mathrm{T} ; z=\mathrm{R}_{0}[y]$ & $\mid \begin{array}{l}401 \text { If } \exists M \neq M^{\prime} \in \mathcal{L}, H^{P}(M)= \\
H^{P}\left(M^{\prime}\right)\end{array}$ \\
\hline Else if $\mathrm{R}_{2}[y] \neq \perp \wedge(y, M) \notin \mathrm{E}$ & $402 \quad$ then bad $=\mathrm{T}$, Finish () \\
\hline then $\mathrm{Bad}=\mathrm{T} ; z=\mathrm{R}_{2}[y]$ & $\begin{array}{l}403 \text { If } \exists M \in \mathcal{L},(y, X) \in \mathrm{E}, X \neq M \\
H^{P}(M)=y\end{array}$ \\
\hline $\mathrm{R}_{0} \longleftarrow(y, z) ;$ return $z ;$ & \begin{tabular}{|l}
404 \\
405 \\
return $\perp$;
\end{tabular} \\
\hline
\end{tabular}

Fig. 4. $G 0$ executes with boxed statements whereas $G 1$ executes without these. Clearly $G 0$ and $G 1$ are identical-until-Bad and whenever $G 1$ set bad true the adversary $B_{A}^{P}$ set also bad true. In this case, Finish() subroutine executes which makes PrA successful. The tuple of simulated oracles of $B_{A}$ is equivalent to $\left(\mathcal{F}, S_{1}, S_{2}\right)$. 